Vol.03/ No. 01

Pages: $21-35$

https://www.irojournals.com/iroiip/

DOI: https://doi.org/10.36548/jiip.2021.1.003

\title{
Simulation of Eye Tracking Control based Electric Wheelchair Construction by Image Segmentation Algorithm
}

\author{
Hadish Habte Tesfamikael, \\ Graduate Assistant, \\ Mainefhi College of Engineering, \\ Asmara, Ministry of Education, Eritrea. \\ hadushabte94@gmail.com
}

Adam Fray,

Junior Telecommunication Engineer, Ministry of Telecommunication, Asmara, Eritrea.

Israel Mengsteab, Junior Power Engineer, Ministry of Defense, Asmara, Eritrea.

\section{Adonay Semere,}

Junior Telecommunication Engineer, Ministry of Telecommunication,

Asmara, Eritrea.

\section{Zebib Amanuel,}

Electrical Installation Supervisor, Ministry of Defense, Asmara, Eritrea.

\begin{abstract}
In this fast-paced world, it is very challenging for the elderly and disabled population to move independently to their desire places at any convenient time. Fortunately, some of the people have good eyesight and physically strong to take care of their survival. Nevertheless, Electric wheelchair (EWC) can provide them a better lifestyle with commendable confidence. At
\end{abstract}


Journal of Innovative Image Processing (JIIP) (2021)

Vol.03/ No. 01

Pages: $21-35$

https://www.irojournals.com/iroiip/

DOI: https://doi.org/10.36548/jiip.2021.1.003

the same time, the hand, head and voice recognition-based EWC meet many limitations. Despite, the eye-tracking-based EWC provides a better smartness in their lifestyle. This research article discusses better accuracy achievement and minimizes the delay response time in the proposed system. The proposed eye-tracking EWC is differed from another existing system with good validation parameters of the controller and it introduces edge detection to identify the eye pupil position in the face. The proposed method includes a PID controller to control the DC motor, which in turn controls the rotation of wheel in EWC. This research article is mainly focused on the costeffectiveness and improvement in the system performance. The display system is mounted in front of the sitting position of EWC users. The camera captures eye pupil position and it determines the direction of the EWC movement by controlling DC motor with the help of a PID controller. When derivative (D) control is used in the proposed system, the system response is quite faster and it reduces the delay time between the user and system reaction. This pupil of eye position is determined by a canny edge detector, which provides good results when compared with other edge detection approaches. Object detection in front of the EWC is an added advantage of the proposed system. The proposed article integrates all the activities and measures the system performance. The proposed model achieves an accuracy of about $90 \%$ and response time is least compared with the existing methods.

Keywords: Electric wheelchair, image processing technique

\section{INTRODUCTION}

Nowadays, the elderly and disable population requires EWC for completing their daily activities at any place and at any time. The primary work of this research paper would be to make their lifestyle simpler and to satisfy them by offering good comfort. On other hand, the operation of complex EWC is a very challenging task for any ill persons [1]. This complexity includes limited direction with 4 ways and not 360 degrees. The eye motion-based EWC does not require any manpower. The camera is placed in front of the use to capture the image and segment eye portion of any human with the help of edge detection techniques [2]. The movement of the eye sends the command for the DC motor to rotate the wheel of the EWC. The advantage of the image processing in EWC is it offers more accuracy for processing any input command. Computer vision 
Journal of Innovative Image Processing (JIIP) (2021)

Vol.03/ No. 01

Pages: $21-35$

https://www.irojournals.com/iroiip/

DOI: https://doi.org/10.36548/jiip.2021.1.003

library is used to train the proposed model for sensing the eye in the face to derive the direction of the wheel. Generally, many research works comprises of the detection of eye movement location from the detected face. Figure 1 shows a simplified version of EWC [3].

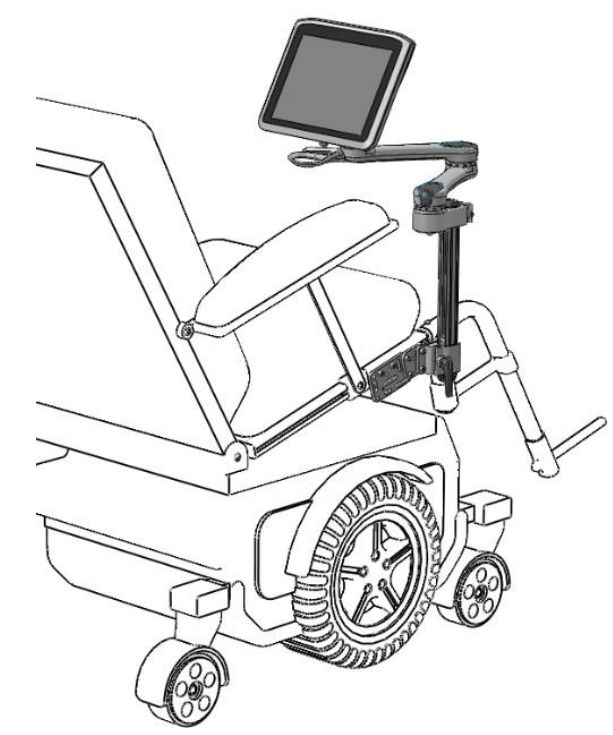

Figure 1 Simplified Picture of Electric Wheelchair

The image processing algorithms are used to detect the edges of the eyes and it helps to determine the position of the eye and assign the direction for the wheel in EWC. The exact movement of the eye pupil can be determined by using an image processing algorithm [4]. Object detection or motion detection techniques are used to detect the eyeball tracking due to their desired direction for the wheelchair. The computer vision libraries of image processing provides many methods to detect the object motion in pattern matching [5]. The faulty direction offers EWC due to many reasons such as some of the person's face and eyes distances are not same as another person. This phenomenon leads to inaccuracy movement of EWC based on wrong identification of eye and face location by same image processing algorithm. The sensor-based eye-tracking system fails due to as above said conditions. Similarly, the head and hand gesture movement system is quite complicated in the sensor-based system [6].

Besides, the voice command system based on EWC fails in open public places due to random noise disturbances and also heavy noise has affected the voice of the person. In public 
Journal of Innovative Image Processing (JIIP) (2021)

Vol.03/ No. 01

Pages: $21-35$

https://www.irojournals.com/iroiip/

DOI: https://doi.org/10.36548/jiip.2021.1.003

places, disabled people should speak or command loudly, which is embarrassing among the crowded people. Moreover, the ill persons will be weak in the course of noisy surrounding places. [7]. The infrared red beam-based eye-tracking system will be very harmful to the patients due to the severity of the ray's content from the beam. The patients may lose their visibility, when they are exposed to same device for a long time. Many research works include a raspberry pi board to monitor and control the complete EWC activity operation [8]. The raspberry pi board controls the incoming and outgoing signals to the board. The motor drive circuit can be controlled by the wheel direction in EWC system. The robot soccer performs in the ground with the color identification of same teammates and color of the ball. This operation can be done by a pure color-coded approach [9]. Therefore, the edge detection of eye pupils will provide better performance of a system with more sophisticated features.

\section{ORGANIZATION OF THE RESEARCH}

The structure of the research article is organized as follows: The existing research works on image processing based electric wheelchair are discussed in section 3. Section 4 presents the proposed methodology of an eye-tracking system for electric wheelchair. The obtained results from the simulation will be discussed in section 5. The conclusion and limitation of the proposed system is discussed in section 6 .

\section{PRELIMINARIES}

The movement of EWC is based on the head movement. Further, a camera is used to detect the patients' requirement and desire for travel direction [10].

The motion of EWC is determined by utilizing the voice commands from wheelchair users. The voice commands are "drive", "stop", right", "left" and so on. The driver is enabled with timevarying concept accordingly. But it suffers from accuracy and delay in start problems [11]. Based on the hand gesture movement, the wheelchair is moving forward, reverse, right and left direction. The system estimation will be computed and arranging between hand gaze movement and direction of wheelchair [12]. The limitation of this model is delay response and the system reacts to the random motion of hand gaze. Electroencephalography-based steering control for an electric wheelchair is a good choice to control. This system can be operated by electrical action from the 
Journal of Innovative Image Processing (JIIP) (2021)

Vol.03/ No. 01

Pages: $21-35$

https://www.irojournals.com/iroiip/

DOI: https://doi.org/10.36548/jiip.2021.1.003

brain through the conductors connected with the scalp of the head [13]. EWC is operated by patients, while no persons are available in the clinical area due to more crowd. In the mid-level of injuries, patients can drive EWC on their own. In voice command, EWC suffers the problems when the patients have some respiratory problems [14]. But the eye-tracking control system of EWC utilizes the camera in the front to capture the activities of the person and based on the determination, EWC will move.

Recently, the eye-tracking based controlled system is considered as a unique control for EWC. Investigation on the eye-tracking control of EWC is a tremendous area in research and solve easily many impairments created by the system. The design of artificial intelligence for eyetracking based EWC is a great advantage to sort out fewer accuracy problems of the devices [15]. Besides, random people can interfere with the camera and it can be destructed the direction of the EWC. Therefore, the system security is very challenging in the existing research works [16]. But our proposed techniques have used pupil tracking goggles connected with the processor to arrange the direction based on their desired direction. The iris of the eye will be an authorized note to the corresponding person. Therefore, this research work can minimize or fix the problem of random people interference. The accuracy and fast response is a matter in eye-tracking-based EWC to improve it. Many research work [17] [18] discusses the limitations of eye-controlled EWC systems and also provides the solution. The author [20] proposes a camera set up to track the eye direction and created the commands based on the system output. The controlling techniques suffer in the response time and accuracy in the performance metrics of the system. The system can be operated by any disabled person except patients, who are unable to move their pupil of the eye. This EWC can move in 4 directions with a limited direction of the rotation of the wheel in the chair [21]. Our research work is directed towards pupil tracking with the authorized iris of the eye is to reduce many problems that arise during real-time operation. They were succeeding in the good system response time for EWC. The existing methods are encouraging the camera quality and restricted descript to sort out the problems. But they fail in many circumstances during the operation of EWC [22]. The Italian group of researchers suggesting the solution of that camera lens with reflected coating for the EWC users. This approach can get high accuracy and good resolution in time.

This approach is working well in lens adjustment in camera and it is also implemented in the proposed system. This non-invasive method of lens adjustment is providing well system 
Journal of Innovative Image Processing (JIIP) (2021)

Vol.03/ No. 01

Pages: $21-35$

https://www.irojournals.com/iroiip/

DOI: https://doi.org/10.36548/jiip.2021.1.003

inaccuracy. This approach is improving the lifestyle quality of the impaired people by providing them a good confidence range and interact themselves with society. The proposed method can provide some intelligence for obstacle detection and this will improve the performance of the existing system.

\section{METHODOLOGIES}

The fundamental idea of our proposed model is the eye tracking-based construction of EWC. The eye pupil is detected by the Haar cascade algorithm to provide good results for enhancing the overall performance of the system [23]. The camera setup in EWC is capturing the face of the person, who uses the wheelchair. The proposed algorithm is initiated to find the facial features and it is confirmed with the original database. According to the proposed algorithm, the face region is detected from the captured images with the specific area. If there is more than one face in a display picture, the proposed system will only respond for the authorized face.

\subsection{Proposed System Overview}

Figure 2 shows the essential parts of proposed system. The movements of the wheel are based on the motor drive receives the commands from camera output through the microcontroller. Also, the obstacle can be detected by using an ultrasonic sensor, which is mounted on the wheelchair for faster detection. If any obstacle comes near to the wheelchair, the raspberry pi sends signal to the driving circuit of the motor to stop further action. The proposed system is a very costeffective and high performance-based eye-tracking control. A better response time can be obtained during the procedure. The proposed system performs well in identifying a human face, accurately locating eye and pupil feature in the face, color to grey conversion, image filtering, pattern recognition, and removal of extra noise that appears in the region of interest of the image.

ISSN: 2582-4252 (online) 
Journal of Innovative Image Processing (JIIP) (2021)

Vol.03/ No. 01

Pages: $21-35$

https://www.irojournals.com/iroiip/

DOI: https://doi.org/10.36548/jiip.2021.1.003

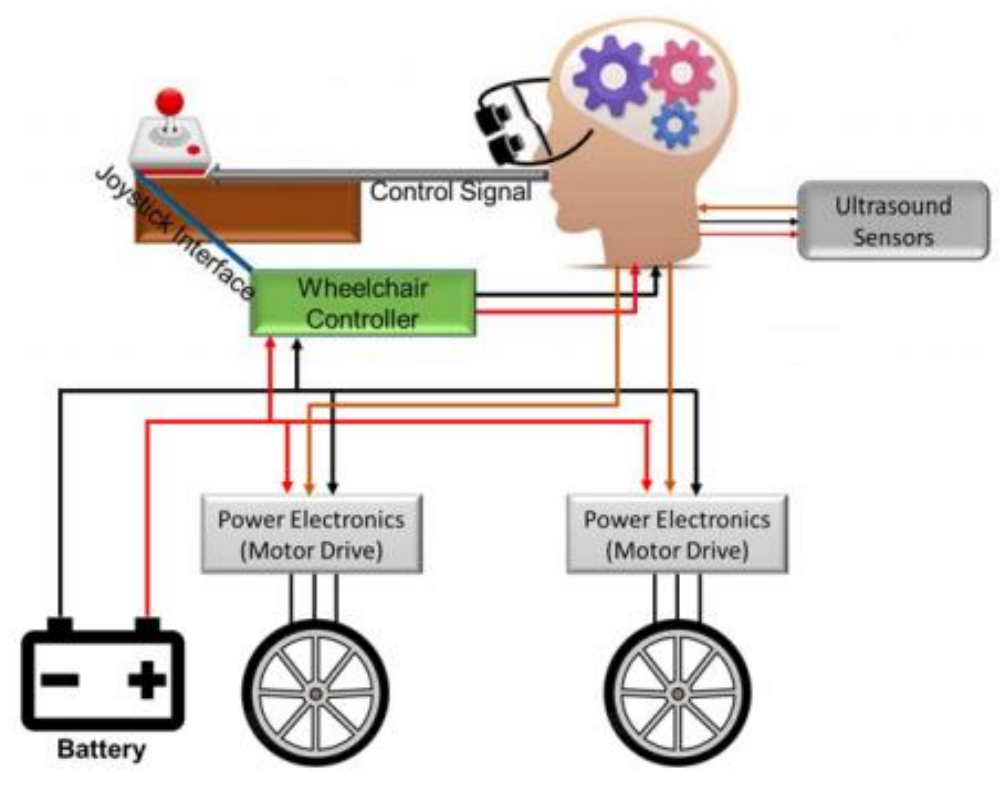

Figure 2 Proposed system arrangement setup

\subsubsection{DC motor Control section}

In the proposed system, DC motor is used to convert electrical signal to mechanical movement. Generally, DC motor is operating in a stationary field with an armature winding of the insulated one. The windings are having multiple turns and adjacent current ways. The end of this winding wire is connected to the commutator, which is used to energize the coil for obtaining rotational power from the battery. The battery is properly connected to the whole system function. The motor drives its rotor based on camera output, which is having various direction movements.

\subsubsection{Image Processing section}

The primary section of proposed system is the image processing section to capture the image. The captured image is detecting the face and pupil of an eye by using edge detection techniques. Depends on the position of the eye, the raspberry pi will react and provides the signal to a motor unit of the EWC system. In the image processing section, there are three subsections to complete our proposed model. 
Journal of Innovative Image Processing (JIIP) (2021)

Vol.03/ No. 01

Pages: $21-35$

https://www.irojournals.com/iroiip/

DOI: https://doi.org/10.36548/jiip.2021.1.003

\section{Canny Edge Detection}

The edges are identified through a canny operator based on pixel intensity changes to draw the boundary. First, the images are enhanced with filtering techniques and it is detecting the edges of the object with pixel intensity in the image by using the threshold method [24].

\section{Haar cascade classifier}

After detecting the face in the image, a haar cascade classifier is used to detect the eye in a better way. Initially, the system starts to capture an image by taking snapshots of the streaming video at a certain time interval. Here, the adjustment of the period between the consecutive snapshots enables the system to minimize the unintentional jerks caused by the involuntary movement of the eye. After the image acquisition process, the implementation of image processing techniques particularly the viola john's algorithm in a cascaded configuration is followed to first detect the face and then the eye. Consequently, the results of the image processing provide prior control commands of the overall system by checking whether the eye (either left or right) is opened or not. If the eye is opened, then it is followed by the pupil tracking algorithm otherwise the system again starts to detect face and eye until it is forwarded by the opening act. At this stage of pupil tracking, the position of the pupil in a coordinate format is obtained to generate the left, right, stop and forward control commands. Once either left or right command is obtained, then the system gets turned to that position accordingly. After the respective commands (either left or right) are executed, the system is pointing in the forward direction whereby the obstacle avoidance algorithm is carried out. When the wheelchair starts and is commanded to move with the help of eye movements, it will detect obstacles and measure the distance in a cycle, make judgment and take necessary action. Here is how the obstacle avoidance procedure work: when the distance measured to the obstacle is less than the given threshold, then the wheelchair will be stopped. After the obstacle avoidance function is completed, the process is again returned to the start of the flow chart so that the users can govern the wheelchair by themselves. By now the pupil tracking algorithm will gain control and the previously discussed flowchart is done in the same manner.

ISSN: 2582-4252 (online) 
Journal of Innovative Image Processing (JIIP) (2021)

Vol.03/ No. 01

Pages: 21-35

https://www.irojournals.com/iroiip/

DOI: https://doi.org/10.36548/jiip.2021.1.003

\section{Hough Transform}

Since the shape of our pupil is circular, Hough transform technique is used to detect the exact shape. First, the image is smoothed to reduce the amount of noise. Then, an edge recognition procedure is implemented. The proposed first-derivative Canny filters are used to determine the edges and edge directions. After some testing, it has also added a second-derivative filter, which is connected with the Canny filters, which offers a more robust way for edge recognition.

\section{RESULTS DISCUSSION}

Eye pupil center localization is the stage we have already detected the pupil as circular shape using the Hough transform. This one is the most difficult section in our proposed methodology and important to calibrate it. Figure 3 is showing a detailed Simulink construction model for DC motor.

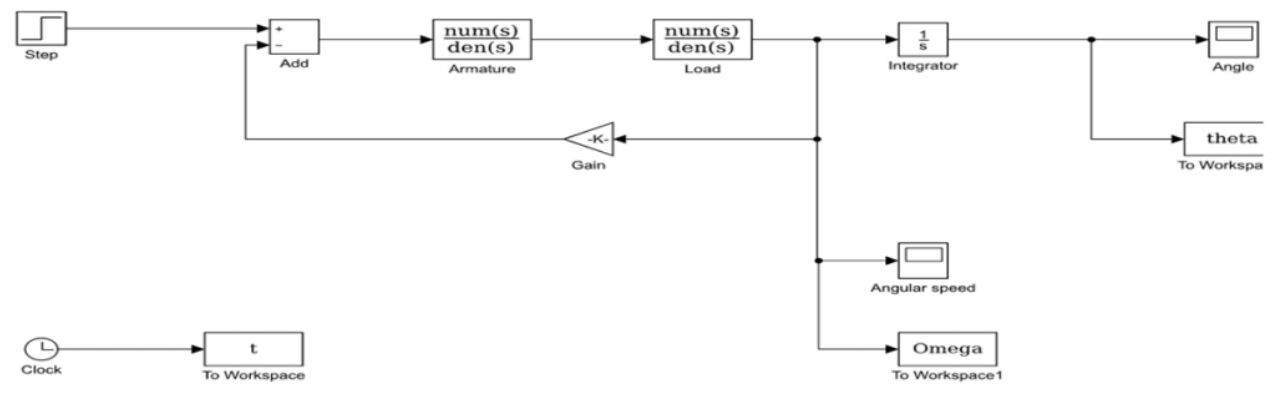

Figure 3 Detailed Simulink Construction of DC Motor

This winding coil is energized with an armature coil for a power supply. The shaft encoders are used to set the direction of the rotor based on its output. The output result of speed vs. time is showing in figure 4.

ISSN: 2582-4252 (online) 
Journal of Innovative Image Processing (JIIP) (2021)

Vol.03/ No. 01

Pages: $21-35$

https://www.irojournals.com/iroiip/

DOI: https://doi.org/10.36548/jiip.2021.1.003

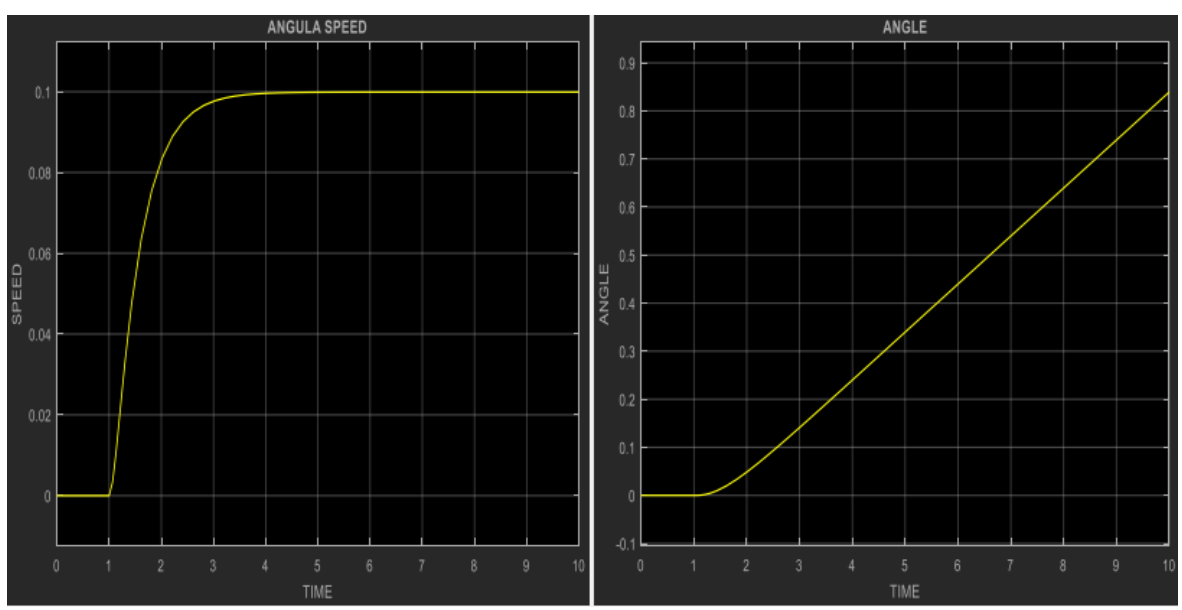

Figure 4 Simulink Output of Settling Time

As per the expectation, proposed system can detect the face and eye of any person, who is standing in front of the camera. Though it is possible to make the system detect either single face or multi-face for the sake of illustration shown by taking the photo of all our group members. Thus, as shown below the faces and eyes are detected. The multiple face detection is shown in figure 5 .

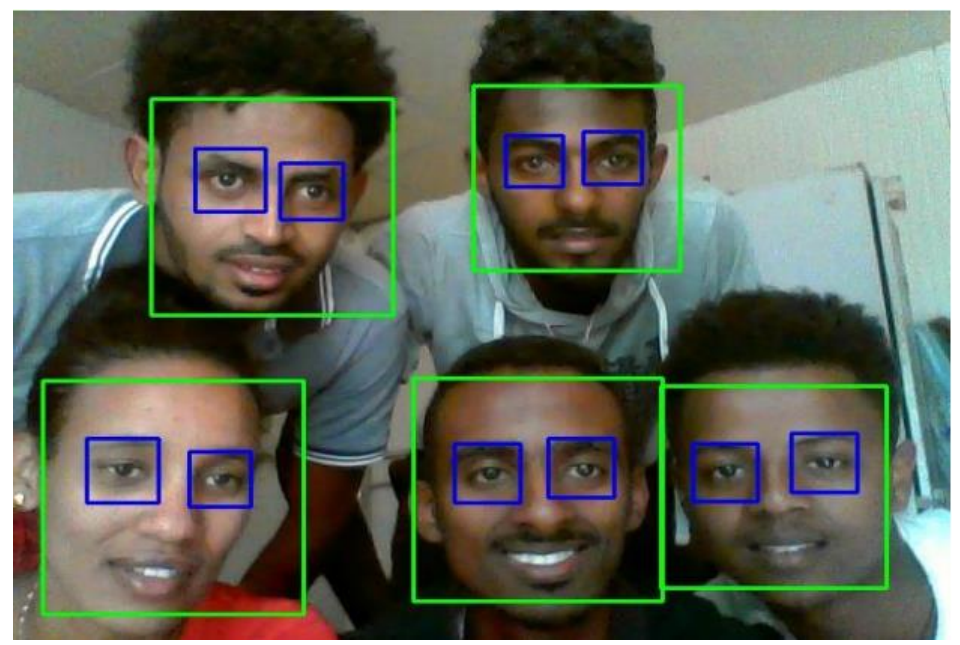

Figure 5 Multiple Face detection by Haar cascade classifier technique

Hereafter, detecting the pupil centroid $(\mathrm{Cx}, \mathrm{Cy})$, continuous checking of the centroids in each snapshot is done. Thus, the spatial coordinate of the pupil centroid relative to the reference origin (left top corner of the image) is taken as left, right, forward, and stop command. 
Journal of Innovative Image Processing (JIIP) (2021)

Vol.03/ No. 01

Pages: $21-35$

https://www.irojournals.com/iroiip/

DOI: https://doi.org/10.36548/jiip.2021.1.003

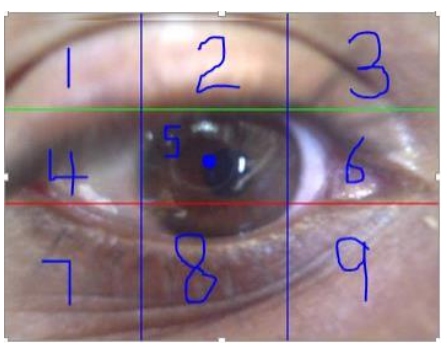

Figure 6 Segmentation in region of eye

Hereafter, it is the stage of localizing the center of the circle. Localizing the center of the circle is done by finding the centroid of the circle. Further, the centroid is typically determined by calculating the image moment of the pupil. The input data from the face to camera, where it is connected with knowledgeable devices. The real-time segmentation of the region of the eye is shown in figure 6.
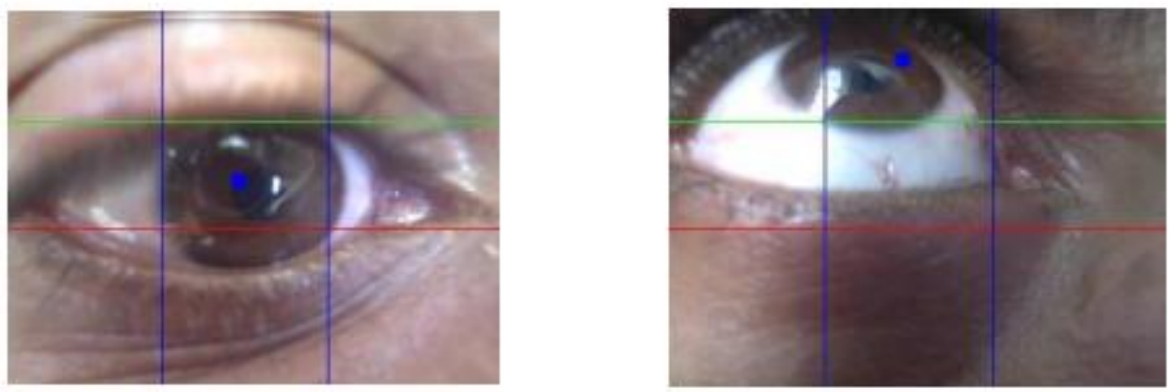

Figure 7 Center and up Positions of eye pupil

Hereafter, the pupil is detected and assignment of its position to the spatial coordinate is done to distinguish the left, right, front, and stop region of coordinates. Initially, the whole image size of the eye is divided into 9 separate regions. The direction is determined by the center box, where details of the eye is showing in Figures 7, 8 and 9. 
Journal of Innovative Image Processing (JIIP) (2021)

Vol.03/ No. 01

Pages: 21-35

https://www.irojournals.com/iroiip/

DOI: https://doi.org/10.36548/jiip.2021.1.003

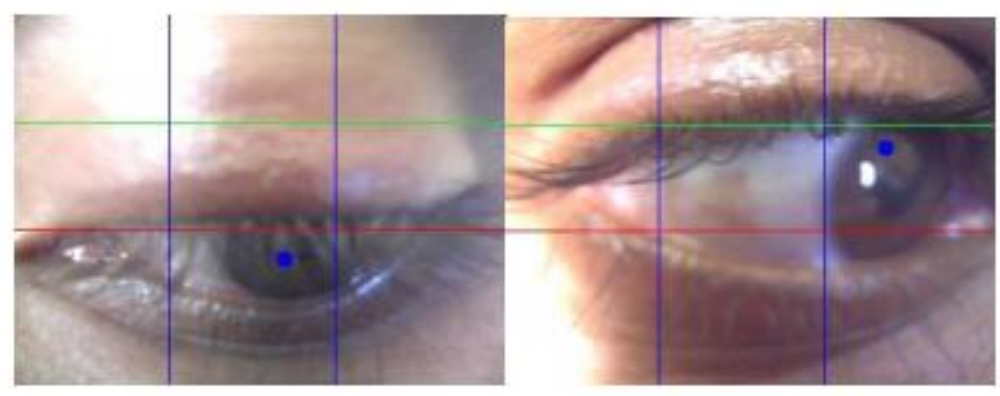

Figure 8 Down and Right Positions of eye pupil

And accordingly, the coordinate of the current pupil position is taken by tracing its location in these separate regions.

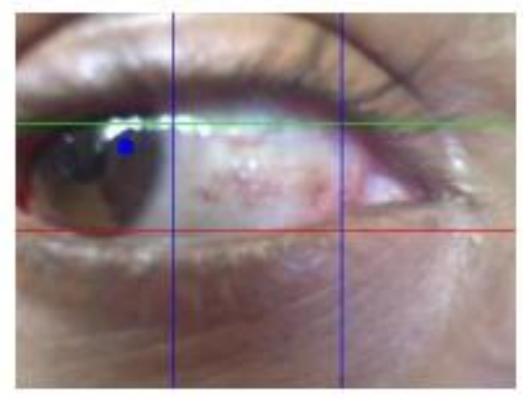

Figure 9 Left Position of eye pupil

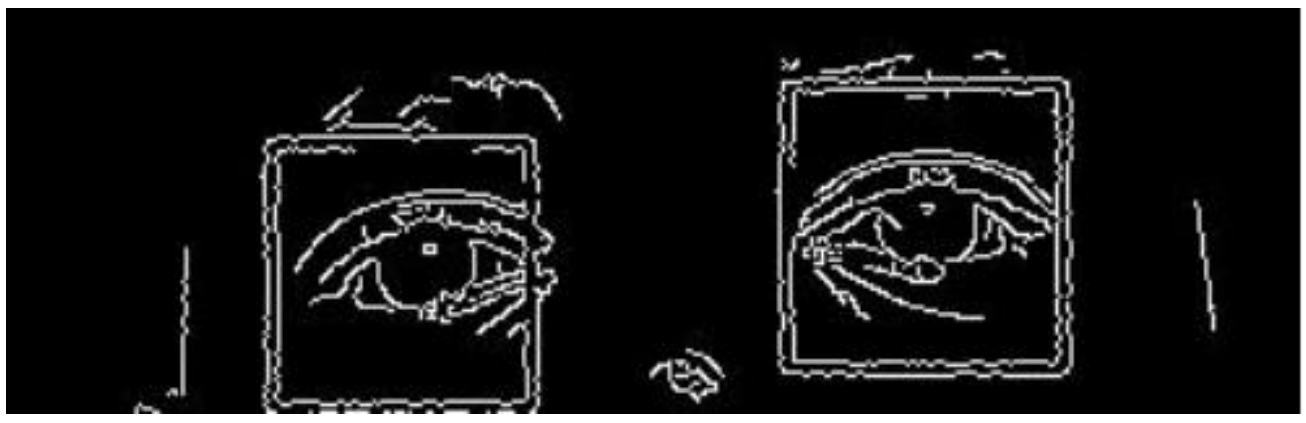

Figure 10 Results Obtained from Canny Edge Detector

Figure 10 shows the obtained results from canny edge detector. 
Journal of Innovative Image Processing (JIIP) (2021)

Vol.03/ No. 01

Pages: $21-35$

https://www.irojournals.com/iroiip/

DOI: https://doi.org/10.36548/jiip.2021.1.003

\section{CONCLUSION}

Thus, eye-tracking controlled wheelchair has been well constructed with image processing concept. The prototype of proposed model has assembled and tested the response time and accuracy of the developed system. Both the measuring units are good enough to achieve our goal of our proposed model. The system consists of an eye-tracking webcam, microcontroller, DC, and servo motors, image processing unit, and associated circuits to design the proposed model. The proposed image processing algorithm is developed by python programming language, which controls the wheels in EWC without much delay. Even though, the proposed concept is most helpful for physically disabled persons, it also has some drawbacks like during night time or dark time, the captured image is not clear and it will be enhanced in future. Except for these problems, the proposed system works flawlessly. Also, the obstacle is finding a forwarding direction to minimize the cost in a more reliable way.

\section{REFERENCES}

[1] J. Kim et al., "The Tongue Enables Computer and Wheelchair Control for People with Spinal Cord Injury”, Science Translational Medicine, Vol 5, Issue 213, 27 November 2013.

[2] S. D. Suryawanshi, J. S. Chitode and S. S. Pethakar, "Voice Operated Intelligent Wheelchair", International Journal of Advanced Research in Computer Science and Software Engineering (IJARCSSE), Volume 3, Issue 5, pp. 487 - 490, May, 2013

[3] S. Paulose, M.P.F. Anooda, G. Mohan, M. S. Sajana and K. A. Anupama, "Automatic Wheelchair using Gesture Recognition Along with Room Automation", Transactions on Engineering and Sciences, Vol. 2, Issue 5, pp. 40 - 43, May 2014.

[4] Zandi, A.S.; Quddus, A.; Prest, L.; Comeau, F.J.E. Non-Intrusive Detection of Drowsy Driving Based on Eye Tracking Data. Transp. Res. Rec. J. Transp. Res. Board 2019, 2673, 247-257. [CrossRef]

[5] Zhang, J.; Yang, Z.; Deng, H.; Yu, H.; Ma, M.; Xiang, Z. Dynamic Visual Measurement of Driver Eye Movements. Sensors 2019, 19, 2217. [CrossRef] [PubMed]

[6] Strobl, M.A.R.; Lipsmeier, F.; Demenescu, L.R.; Gossens, C.; Lindemann, M.; De Vos, M. Look me in the eye: Evaluating the accuracy of smartphone-based eye tracking for potential 
Journal of Innovative Image Processing (JIIP) (2021)

Vol.03/ No. 01

Pages: 21-35

https://www.irojournals.com/iroiip/

DOI: https://doi.org/10.36548/jiip.2021.1.003

application in autism spectrum disorder research. Biomed. Eng. Online 2019, 18, 51. [CrossRef] [PubMed]

[7] Shishido, E.; Ogawa, S.; Miyata, S.; Yamamoto, M.; Inada, T.; Ozaki, N. Application of eye trackers for understanding mental disorders: Cases for schizophrenia and autism spectrum disorder. Neuropsychopharmacol. Rep. 2019, 39, 72-77. [CrossRef]

[8] Cruz, R.; Souza, V.; Filho, T.B.; Lucena, V. Electric Powered Wheelchair Command by Information Fusion from Eye Tracking and BCI. In Proceedings of the 2019 IEEE International Conference on Consumer Electronics (ICCE), Las Vegas, NV, USA, 11-13 January 2019.

[9] Jafar, F.; Fatima, S.F.; Mushtaq, H.R.; Khan, S.; Rasheed, A.; Sadaf, M. Eye Controlled Wheelchair Using Transfer Learning. In Proceedings of the 2019 International Symposium on Recent Advances in Electrical Engineering (RAEE), Islamabad, Pakistan, 28-29 August 2019. [10] Deshpande, S.; Adhikary, S.D.; Arvindekar, S.; Jadhav, S.S.; Rathod, B. Eye Monitored Wheelchair Control for People Suffering from Quadriplegia. Univers. Rev. 2019, 8, 141-145.

[11] Majaranta, P.; Bulling, A. Eye Tracking and Eye-Based Human-Computer Interaction. In Human-Computer Interaction Series; Springer Science and Business Media LLC: London, UK, 2014; pp. 39-65.

[12] Harezlak, K.; Kasprowski, P. Application of eye tracking in medicine: A survey, research issues and challenges. Comput. Med. Imaging Graph. 2018, 65, 176-190. [CrossRef]

[13] Liu, T.-L.; Fan, C.-P. Visible-light wearable eye gaze tracking by gradients-based eye center location and head movement compensation with IMU. In Proceedings of the 2018 IEEE International Conference on Consumer Electronics (ICCE), Las Vegas, NV, USA, 12-14 January 2018.

[14] Robbins, S.; McEldowney, S.; Lou, X.; Nister, D.; Steedly, D.; Miller, Q.S.C.; Bohn, D.D.; Terrell, J.P.; Goris, A.C.; Ackerman, N. Eye-Tracking System Using a Freeform Prism and GazeDetection Light. U.S. Patent 10,228,561, 12 March 2019.

[15] Sasaki, M.; Nagamatsu, T.; Takemura, K. Screen corner detection using polarization camera for cross-ratio based gaze estimation. In Proceedings of the 11th ACM Symposium on Eye Tracking Research \& Applications, Denver, CO, USA, 25-28 June 2019.

[16] Holland, J. Eye Tracking: Biometric Evaluations of Instructional Materials for Improved Learning. Int. J Educ. Pedag. Sci. 2019, 13, 1001-1008.

ISSN: 2582-4252 (online) 
Journal of Innovative Image Processing (JIIP) (2021)

Vol.03/ No. 01

Pages: $21-35$

https://www.irojournals.com/iroiip/

DOI: https://doi.org/10.36548/jiip.2021.1.003

[17] Rabhi, Y.; Mrabet, M.; Fnaiech, F. A facial expression controlled wheelchair for people with disabilities. Comput. Methods Programs Biomed. 2018, 165, 89-105. [CrossRef]

[18] Arai, K.; Mardiyanto, R.; Nopember, K.I.T.S. A Prototype of ElectricWheelchair Controlled by Eye-Only for Paralyzed User. J. Robot. Mechatron. 2011, 23, 66-74. [CrossRef]

[19] Mani, N.; Sebastian, A.; Paul, A.M.; Chacko, A.; Raghunath, A. Eye controlled electric wheel chair. Int. J. Adv. Res. Electr. Electron. Instrum. Eng. 2015, 4. [CrossRef]

[20] Gautam, G.; Sumanth, G.; Karthikeyan, K.; Sundar, S.; Venkataraman, D. Eye movement based electronic wheel chair for physically challenged persons. Int. J. Sci. Technol. Res. 2014, 3, 206-212.

[21] Patel, S.N.; Prakash, V.; Narayan, P.S. Autonomous camera based eye controlled wheelchair system using raspberry-pi. In Proceedings of the 2015 International Conference on Innovations in Information, Embedded and Communication Systems (ICIIECS), Coimbatore, India, 19-20 March 2015.

[22] Chacko, J.K.; Oommen, D.; Mathew, K.K.; Sunny, N.; Babu, N. Microcontroller based EOG guided wheelchair. Int. J. Med. Health Pharm. Biomed. Eng. 2013, 7, 409-412.

[23] Hickson, S.; Dufour, N.; Sud, A.; Kwatra, V.; Essa, I. Eyemotion: Classifying Facial Expressions in VR Using Eye-Tracking Cameras. In Proceedings of the 2019 IEEE Winter Conference on Applications of Computer Vision (WACV), Waikoloa Village, HI, USA, 7-11 January 2019.

[24] C. -. Tyan and P. P. Wang, "Image processing-enhancement, filtering and edge detection using the fuzzy logic approach," [Proceedings 1993] Second IEEE International Conference on Fuzzy Systems, San Francisco, CA, USA, 1993, pp. 600-605 vol.1, doi: 10.1109/FUZZY.1993.327420.

ISSN: 2582-4252 (online) 